International Journal of Algebra, Vol. 1, 2007, no. 9, 421 - 428

\title{
On Two Identities of the Rank of Matrix Product
}

\author{
Jun-qing Wang and Chang-zhou Dong ${ }^{1}$ \\ Mathematics Department \\ Tianjin Polytechnic University \\ Tianjin, 300160, P. R. China
}

\begin{abstract}
We proved two important identities of the rank of the product of matrixes by using block matrixes and system of solutions of reference of homogeneous linear equations and give some useful equalities and inequalities of rank of the product of the matrixes base on these identities.
\end{abstract}

\section{Mathematics Subject Classification: 15A24}

Keywords: block matrix; system of solutions of reference; rank of matrix; identity

\section{Introduction}

The rank of matrix is a very pointed and useful notion, there are many equalities and inequalities about the rank of matrix, such as well-known Sylvester inequality

$$
r(A B) \geq r(A)-r(B)-n .
$$

and Frobenius inequality

$$
r(A B C) \geq r(A B)-r(B C)-r(B) .
$$

In this study, we proved two important identities of the rank of the product of matrix by using block matrix and system of solutions of reference of homogeneous linear equations, by using block matrix we can direct derive Sylvester inequality and Frobenius inequality.

\footnotetext{
${ }^{1}$ Corresponding author. e-mail: dongchangzh@sina.com
} 


\section{Lemmas}

Lemma 1. Let $V$ is vector space with dimension $n$ of number filed $P$, $\alpha_{1}, \alpha_{2}, \ldots, \alpha_{n}$ is a set of basis of $V, \beta_{1}, \beta, \ldots, \beta_{m}$ is a random set of vector of $V, W=L\left(\beta_{1}, \beta_{2}, \ldots, \beta_{m}\right)$, the matrix of $\sigma$ act on basis $\alpha_{1}, \alpha_{2}, \ldots, \alpha_{n}$ is $A$, where $\sigma$ is linear transformation of $V$. That is

$$
\sigma\left(\alpha_{1}, \alpha_{2}, \ldots, \alpha_{n}\right)=\left(\alpha_{1}, \alpha_{2}, \ldots, \alpha_{n}\right) A
$$

Let

$$
\left(\beta_{1}, \beta_{2}, \ldots, \beta_{m}\right)=\left(\alpha_{1}, \alpha_{2}, \ldots, \alpha_{n}\right) B, B \in P^{n \times m}
$$

Then

$$
\operatorname{dim} \sigma(W)=r(A B)
$$

Proof. Using

$$
W=L\left(\beta_{1}, \beta_{2}, \ldots, \beta_{m}\right), \sigma(W)=L\left(\sigma \beta_{1}, \sigma \beta, \ldots, \sigma \beta_{m}\right)
$$

and

$$
\left(\sigma \beta_{1}, \sigma \beta_{2}, \ldots, \sigma \beta_{m}\right)=\sigma\left(\alpha_{1}, \alpha_{2}, \ldots, \alpha_{n}\right) B=\left(\alpha_{1}, \alpha_{2}, \ldots, \alpha_{n}\right) A B
$$

So

$$
\operatorname{dim} \sigma(W)=\left(\sigma \beta_{1}, \sigma \beta_{2}, \ldots, \sigma \beta_{m}\right)=r(A B)
$$

Lemma 2. let $V$ is vector space with dimension $n$ of number field $P, W$ is subspace of $V, \sigma$ is linear transformation of $V$.

Then

$$
\operatorname{dim} \sigma(W)=\operatorname{dim} W-\operatorname{dim}\left(\sigma^{-1}(0) I W\right)=\operatorname{dim}\left(W+\sigma^{-1}(0)\right)-\operatorname{dim} \sigma^{-1}(0)
$$

Proof. Suppose $\operatorname{dim} W=s, \operatorname{dim}\left(\sigma^{-1}(0) I W\right)=r$, take a set of basis of $\sigma^{-1}(0) I W$ is $\alpha_{1}, \alpha_{2}, \ldots, \alpha_{r}$, and extend it into $\alpha_{1}, \alpha_{2}, \ldots, \alpha_{r}, \beta_{1}, \beta_{2}, \ldots, \beta_{s-r}$, where $\alpha_{1}, \alpha_{2}, \ldots, \alpha_{r}, \beta_{1}, \beta_{2}, \ldots, \beta_{s-r}$, is a group of basis of $W$.

Then

$$
\sigma(W)=L\left(\sigma \alpha_{1}, \sigma \alpha_{2}, \ldots, \sigma \alpha_{r}, \sigma \beta_{1}, \sigma \beta_{2}, \ldots, \sigma \beta_{s-r}\right)
$$

Since

$$
\sigma \alpha_{i}=0(i=1,2, \ldots, r)
$$

So

$$
\sigma(W)=L\left(\sigma \beta_{1}, \sigma \beta_{2}, \ldots, \sigma \beta_{s-r}\right)
$$


Let $\sum_{i=1}^{s-r} k_{i} \sigma \beta_{i}=0$, thus $\sigma\left(\sum_{i=1}^{s-r} k_{i} \beta_{i}\right)=0$, that is $\sum_{i=1}^{s-r} k_{i} \beta_{i} \in \sigma^{-1}(0)$, so $\sum_{i=1}^{s-r} k_{i} \beta_{i}$ can be linear expression by $\alpha_{1}, \alpha_{2}, \ldots, \alpha_{r}$, suppose $\sum_{i=1}^{s-r} k_{i} \beta_{i}=\sum_{i=1}^{r} k_{i} \alpha_{i}$, that is

$$
\sum_{i=1}^{s-r} k_{i} \beta_{i}-\sum_{i=1}^{r} k_{i} \alpha_{i}=0
$$

By $\alpha_{1}, \alpha_{2}, \ldots, \alpha_{r}, \beta_{1}, \beta_{2}, \ldots, \beta_{s-r}$, is linearly independent ,then $k_{i}=0(i=$ $0,1,2 \ldots, s-r)$, hence $\sigma \beta_{1}, \sigma \beta_{2}, \ldots, \sigma \beta_{s-r}$ is linearly independent, so

$$
\operatorname{dim} \sigma(W)=s-r=\operatorname{dim} W-\operatorname{dim}\left(\sigma^{-1}(0) I W\right)
$$

We can derive by lemma 1

$$
\operatorname{dim} W+\operatorname{dim} \sigma^{-1}(0)=\operatorname{dim}\left(\sigma^{-1}(0) I W\right)+\operatorname{dim}\left(W+\sigma^{-1}(0)\right)
$$

That is

$$
\operatorname{dim} W-\operatorname{dim}\left(\sigma^{-1}(0) I W\right)=\operatorname{dim}\left(W+\sigma^{-1}(0)\right)-\operatorname{dim} \sigma^{-1}(0)
$$

Therefore,we obtain

$$
\operatorname{dim} \sigma(W)=\operatorname{dim} W-\operatorname{dim}\left(\sigma^{-1}(0) I W\right)=\operatorname{dim}\left(W+\sigma^{-1}(0)\right)-\operatorname{dim} \sigma^{-1}(0)
$$

Lemma 3. Suppose $A_{i} \in P^{m \times n}, i=1,2, \ldots, k$. Then

$$
\left(\begin{array}{cccc}
A_{1} & & & 0 \\
& A_{2} & & \\
& & \ddots & \\
* & & & A^{k}
\end{array}\right) \geq \sum_{i=1}^{k} r\left(A_{i}\right)
$$

Lemma 4. Suppose $A_{m \times n}, B_{m \times t}$ is matrix.

Then

$$
r(A, B) \geq \max (r(A), r(B)) .
$$

\section{Theorem}

Theorem 1. Suppose $A \in P^{m \times n}, B \in P^{n \times m}$. Then

$$
r(A B)=r(A)+r\left(B, \eta_{1}, \eta_{2}, \ldots, \eta_{n-r(A)}\right)-n
$$


Where $\eta_{1}, \eta_{2}, \ldots, \eta_{n-r(A)}$ is system of solutions of reference of homogeneous linear equations $A X=0$.

Proof. (1) $A$ is square matrix with order $n$, let $V$ is vector space with dimension $n$ of number filed $P, \alpha_{1}, \alpha_{2}, \ldots, \alpha_{n}$ is a set of basis of $V$, suppose the matrix of $\sigma$ act on basis $\alpha_{1}, \alpha_{2}, \ldots, \alpha_{n}$ is $A$, where $\sigma$ is linear transformation of $A$, that is ,

$$
\sigma\left(\alpha_{1}, \alpha_{2}, \ldots, \alpha_{n}\right)=\left(\alpha_{1}, \alpha_{2}, \ldots, \alpha_{n}\right) A
$$

Suppose $\left(\beta_{1}, \beta_{2}, \ldots, \beta_{m}\right)=\left(\alpha_{1}, \alpha_{2}, \ldots, \alpha_{n}\right) B, B \in P^{n \times m}, W=L\left(\beta_{1}, \beta_{2}, \ldots, \beta_{m}\right)$ We can derive by lemma 2 ,

$$
r(A B)=\operatorname{dim} \sigma(W)=\operatorname{dim}\left(W+\sigma^{-1}(0)\right)-\operatorname{dim} \sigma^{-1}(0)
$$

And $\operatorname{dim} \sigma^{-1}(0)=n-r(A)$, so the basis of $\sigma^{-1}(0)$ is

$$
\left(\alpha_{1}, \alpha_{2}, \ldots, \alpha_{n}\right) \eta_{1},\left(\alpha_{1}, \alpha_{2}, \ldots, \alpha_{n}\right) \eta_{2} \ldots,\left(\alpha_{1}, \alpha_{2}, \ldots, \alpha_{n}\right) \eta_{n-r(A)}
$$

Where $\eta_{1}, \eta_{2}, \ldots, \eta_{n-r(A)}$ is system of solutions of reference of homogeneous linear equations $A X=0$, then

$$
\begin{aligned}
\sigma^{-1}(0) & =\left\{\xi \mid A\left(\begin{array}{c}
x_{1} \\
x_{2} \\
\vdots \\
x_{n}
\end{array}\right)=0\right\} \\
& =L\left(\left(\alpha_{1}, \alpha_{2}, \ldots, \alpha_{n}\right) \eta_{1}, \ldots,\left(\alpha_{1}, \alpha_{2}, \ldots, \alpha_{n}\right) \eta_{n-r(A)}\right) \\
& =L\left(\left(\alpha_{1}, \alpha_{2}, \ldots, \alpha_{n}\right)\left(\eta_{1}, \eta_{2}, \ldots, \eta_{n-r(A)}\right)\right.
\end{aligned}
$$

so

$$
\begin{aligned}
W+\sigma^{-1}(0) & =L\left(\beta_{1}, \beta_{2}, \ldots, \beta_{m},\left(\alpha_{1}, \alpha_{2}, \ldots, \alpha_{n}\right)\left(\eta_{1}, \eta_{2}, \ldots, \eta_{n-r(A)}\right)\right) \\
& =L\left(\left(\alpha_{1}, \alpha_{2}, \ldots, \alpha_{n}\right) B,\left(\alpha_{1}, \alpha_{2}, \ldots, \alpha_{n}\right)\left(\eta_{1}, \eta_{2}, \ldots, \eta_{n-r(A)}\right)\right) \\
& =L\left(B, \eta_{1}, \eta_{2}, \ldots, \eta_{n-r(A)}\right)
\end{aligned}
$$

Thus

$$
\begin{aligned}
r(A B) & =r(A)+\operatorname{dim} L\left(B, \eta_{1}, \eta_{2}, \ldots, \eta_{n-r(A)}\right)-n \\
& =r(A)+r\left(B, \eta_{1}, \eta_{2}, \ldots, \eta_{n-r(A)}\right)-n
\end{aligned}
$$

(2) $A \in P^{m \times n}$,when $s<n$, let $A_{1}=\left(\begin{array}{c}A \\ 0\end{array}\right)_{m \times n}, A_{1} B=\left(\begin{array}{c}A B \\ 0\end{array}\right)_{m \times n}$, , 
Then $r\left(A_{1} B\right)=r(A B)$ fand $A X=0$ is same solution to $A_{1} X=0$, so equality (1) is right. When $s>n$, let $A_{1}=(A, 0)_{s \times s}, B_{1}=\left(\begin{array}{c}B \\ 0\end{array}\right)_{s \times m}$, then $r\left(A_{1} B_{1}\right)=r(A B)$, and the system of solution of reference of $(\mathrm{A}, 0) \mathrm{X}=0$ is

$$
\begin{aligned}
\eta_{1}^{\prime} & =\left(\begin{array}{c}
\eta_{1} \\
0 \\
\vdots \\
0
\end{array}\right), \ldots, \eta_{n-r(A)}^{\prime}=\left(\begin{array}{c}
\eta_{n-r(A)} \\
0 \\
\vdots \\
0
\end{array}\right), \\
\eta_{n-r(A)+1}^{\prime} & =\left(\begin{array}{c}
\eta_{n-r(A)+1} \\
1 \\
0 \\
\vdots \\
0
\end{array}\right), \ldots, \eta_{s-r(A)}^{\prime}=\left(\begin{array}{c}
e t a_{s-r(A)} \\
0 \\
\vdots \\
0 \\
1
\end{array}\right)
\end{aligned}
$$

So

$$
r\left(B_{1}, \eta_{1}^{\prime}, \eta_{2}^{\prime}, \ldots, \eta_{n-r(A)}^{\prime}\right)=r\left(B_{1}, \eta_{1}, \eta_{2}, \ldots, \eta_{n-r(A)}\right)+s-n
$$

Thus, equality (1) is right.

Consequence 1. Suppose $A_{n \times m}, B_{n \times m}$ is matrix. Then

$$
r(A B)=r(B)+r\left(A^{\prime}, \xi_{1}, \xi_{2} \ldots, \xi_{n-r(B)}\right)+s-n
$$

Where $\xi_{1}, \xi_{2} \ldots, \xi_{n-r(B)}$ is system of solutions of reference of homogeneous linear equations $B^{\prime} X=0$.

In fact, consequence 2 can be derived by $r(A B)=r\left((A B)^{\prime}\right)=r\left(B^{\prime} A^{\prime}\right)$ and lemma 1.

Consequence 2. (Sylvester inequality) Suppose $A_{n \times m}, B_{n \times m}$ is matrix. Then

$$
r(A B) \geq r(A)+r(B)-n .
$$

In fact, consequence 2 can be derived by $r\left(B, \eta_{1}, \eta_{2}, \ldots, \eta_{n-r(A)}\right) \geq r(B)$ and theorem 1.

Consequence 3. Suppose $A \in P^{m \times n}, k$ is positive integer.

Then

$$
r\left(A^{k}\right)=r(A)+\sum_{i=1}^{k-1} r\left(A, \eta_{1}^{(i)}, \eta_{2}^{(i)}, \ldots, \eta_{n-r(A)}^{(i)}\right)-(k-1) n
$$

Where $\left.\eta_{1}^{(i)}, \eta_{2}^{(i)}, \ldots, \eta_{n-r(A)}^{(i)}\right)$ is system of solutions of reference of homogeneous linear equations $A^{i} X=0(i=1,2, \ldots, k-1)$. 
In fact, we can derive by theorem 1 ,

$$
r\left(A^{i}\right)=r\left(A^{i-1}\right)+r\left(A, \eta_{1}^{(i-1)}, \eta_{2}^{(i-1)}, \ldots, \eta_{n-r(A)}^{(i-1)}\right)-n(i=2,3, \ldots, k)
$$

and we can derive consequence 3 by adding those equalities above together.

Consequence 4. Suppose $A \in P^{n \times m}, B \in P^{p \times m}, C \in P^{n \times q}, B$ is column nonsingular matrix,$C$ is row nonsingular matrix.

Then

$$
r(B A)=r(A C)=r(A B C)=r(A)
$$

Proof. Assume system of solutions of reference of homogeneous linear equations of $A^{\prime} X=0$ is $\xi_{1}, \xi_{2} \ldots, \xi_{m-r(A)}$ system of solution of reference of homogeneous linear equations of $A X=0$ is $\eta_{1}, \eta_{2}, \ldots, \eta_{n-r(A)}$, then

$$
\begin{gathered}
r(B A)=r\left(A^{\prime} B^{\prime}\right)=r(A)+r\left(B^{\prime}, \xi_{1}, \xi_{2} \ldots, \xi_{m-r(A)}\right)-m, \\
r(A C)=R(A)+r\left(C, \eta_{1}, \eta_{2}, \ldots, \eta_{n-r(A)}\right)-n
\end{gathered}
$$

Because both $B^{\prime}$ and $C^{\prime}$ is row nonsingular matrix

Hence

$$
\begin{aligned}
& r\left(B^{\prime}, \xi_{1}, \xi_{2} \ldots, \xi_{m-r(A)}\right)=m \\
& r\left(C, \eta_{1}, \eta_{2}, \ldots, \eta_{n-r(A)}\right)=n
\end{aligned}
$$

So

$$
r(B A)=r(A C)=r(A)
$$

Thus

$$
r(B A C)=r(A)
$$

Theorem 2. Suppose $A_{i} \in P^{n \times n}, i=1,2, \ldots, k$. Then

$$
r\left(A_{1}, A_{2}, \ldots, A_{k}\right)=r\left(\begin{array}{ccccc}
A_{1} & & & & 0 \\
I_{n_{1}} & A_{2} & & & \\
& I_{n_{2}} & A_{3} & & \\
& & \ddots & \ddots & \\
0 & & & I_{n_{k-1}} & A_{k}
\end{array}\right)-\sum_{i=1}^{k-1} n_{i}
$$

Proof. we can derive by using general elementary transformation of block matrix 


$$
\begin{aligned}
& \left(\begin{array}{ccccc}
A_{1} & & & & 0 \\
I_{n_{1}} & A_{2} & & & \\
& I_{n_{2}} & A_{3} & & \\
& & \ddots & \ddots & \\
0 & & & I_{n_{k-1}} & A_{k}
\end{array}\right) \\
& \rightarrow \quad\left(\begin{array}{ccccc}
0 & -A_{1} A_{2} & & & \\
I_{n_{1}} & A_{2} & & & \\
& I_{n_{2}} & A_{3} & & \\
& & \ddots & \ddots & \\
& & & I_{n_{k-1}} & A_{k}
\end{array}\right) \\
& \rightarrow \quad\left(\begin{array}{ccccc}
0 & 0 & A_{1} A_{2} A_{3} & & \\
I_{n_{1}} & A_{2} & & & \\
& I_{n_{2}} & A_{3} & & \\
& & \ddots & \ddots & \\
0 & & & I_{n_{k-1}} & A_{k}
\end{array}\right) \\
& \rightarrow \ldots \rightarrow\left(\begin{array}{ccccc}
0 & 0 & \ldots & (-1)^{k-2} A_{1} A_{2} \ldots A_{k-1} & 0 \\
I_{n_{1}} & A_{2} & & & 0 \\
& I_{n_{2}} & A_{3} & & 0 \\
& & \ddots & \ddots & \vdots \\
& & & I_{n_{k-1}} & A_{k}
\end{array}\right) \\
& \rightarrow \quad\left(\begin{array}{ccccc}
0 & 0 & 0 & \ldots & (-1)^{k-1} A_{1} A_{2} \ldots A_{k} \\
I_{n_{1}} & 0 & & & 0 \\
& I_{n_{2}} & 0 & & 0 \\
& & \ddots & \ddots & \vdots \\
& & & I_{n_{k-1}} & 0
\end{array}\right) \\
& =\quad r\left(A_{1}, A_{2}, \ldots, A_{k}\right)+\sum_{i=1}^{k-1} n_{i}
\end{aligned}
$$

Thus $£$ equality (2) is right.

Furthermore, we can represent the rank of matrix multiplication by using the method of organizing simple block matrix from dividing of the product of finite matrix into segment.

The Sylvester inequality of rank of matrix multiplication $r(A B) \geq r(A)+$ 
$r(B)-n$ is important in matrix theorem . By using theorem 2 and the properties of block matrix, we can generalize Sylvester inequality as follows.

Consequence 1. Suppose $A_{i} \in P^{n \times n}, i=1,2, \ldots, k$. Then $r\left(A_{1}\right)+r\left(A_{2}\right)+\ldots+r\left(A_{k}\right)-\sum_{i=1}^{k-1} n_{i} \leq r\left(A_{1}, A_{2}, \ldots, A_{k}\right) \leq \min \left(r\left(A_{1}\right), r\left(A_{2}\right) \ldots, r\left(A_{k}\right)\right)$

Consequence 2. (Frobenius inequality) Suppose $A_{m \times n}, B_{n \times p}, C_{p \times q}$ is matrix.

Then

$$
r(A B C) \geq r(A B) \geq r(B C)-r(B)
$$

\section{Proof.}

$$
r(A B C)=r\left(\begin{array}{cc}
A B & 0 \\
B & B C
\end{array}\right)-r(B) \geq r(A B) \geq r(B C)-r(B)
$$

That is , Frobenius inequality is right .

\section{References}

[1] ]Mathematics And Mechanics Department of Beijing University. Higher Algebra (Version 2 ) [M] Beijing: Higher Education Publisher, 1990.

[2] Huang Yan-zhu, Zhong Shou-ming, Li Zheng-liang, Matrix theory (Version 1) $[\mathrm{M}]$. Beijing: Higher Education Publisher, 2003.

[3] Ni Guo-xi, Common Matrix Theory and Method [M]. Shanghai: Shanghai Science Publisher, 1984.

[4] M. Marcus \& H. Minc, A Survey of Matrix Theory and Matrix Inequalities [M]. Allyn and Bacon, 1964.

Received: March 21, 2007 